\title{
Exact Controllability for Stochastic Schrödinger Equations*
}

\author{
Qi L $\ddot{u ̈}^{\dagger}$
}

\begin{abstract}
This paper is addressed to studying the exact controllability for stochastic Schrödinger equations by two controls. One is a boundary control in the drift term and the other is an internal control in the diffusion term. By means of the standard duality argument, the control problem is converted into an observability problem for backward stochastic Schrödinger equations, and the desired observability estimate is obtained by a global Carleman estimate. At last, we give a result about the lack of exact controllability, which shows that the action of two controls is necessary.
\end{abstract}

2010 Mathematics Subject Classification. Primary 93B05; Secondary 93B07, 93E20, $60 \mathrm{H} 15$.

Key Words. Stochastic Schrödinger equations, exact controllability, observability, Carleman estimate.

\section{Introduction}

Let $T>0, G \subset \mathbb{R}^{n}(n \in \mathbb{N})$ be a given bounded domain with the $C^{2}$ boundary $\Gamma$. Let $\Gamma_{0}$ be a suitable chosen nonempty subset (to be given later) of $\Gamma$. Put $Q \triangleq(0, T) \times G$, $\Sigma \triangleq(0, T) \times \Gamma$ and $\Sigma_{0} \triangleq(0, T) \times \Gamma_{0}$.

Let $\left(\Omega, \mathcal{F},\left\{\mathcal{F}_{t}\right\}_{t \geq 0}, P\right)$ be a complete filtered probability space on which a one dimensional standard Brownian motion $\{B(t)\}_{t \geq 0}$ is defined such that $\left\{\mathcal{F}_{t}\right\}_{t \geq 0}$ is the natural filtration generated by $\{B(t)\}_{t \geq 0}$, augmented by all the $P$-null sets in $\mathcal{F}$. Let $H$ be a Banach space. We denote by $L_{\mathcal{F}}^{2}(0, T ; H)$ the Banach space consisting of all $H$-valued $\left\{\mathcal{F}_{t}\right\}_{t \geq 0}$-adapted processes $X(\cdot)$ such that $\mathbb{E}\left(|X(\cdot)|_{L^{2}(0, T ; H)}^{2}\right)<\infty$; by $L_{\mathcal{F}}^{\infty}(0, T ; H)$ the Banach space consisting of all $H$-valued $\left\{\mathcal{F}_{t}\right\}_{t \geq 0}$-adapted bounded processes; and by $C_{\mathcal{F}}\left([0, T] ; L^{2}(\Omega ; H)\right)$ the Banach space

\footnotetext{
*This work is partially supported by the NSF of China under grant 11101070 and the Fundamental Research Funds for the Central Universities in China under grants ZYGX2012J115.

${ }^{\dagger}$ Université Pierre et Marie Curie-Paris VI UMR 7598, Laboratoire Jacques-Louis Lions, 4, place Jussieu Paris, F-75005 France; School of Mathematical Sciences, University of Electronic Science and Technology of China, Chengdu, 610054, China. E-mail: Iuqi59@163.com.
} 
consisting of all $H$-valued $\left\{\mathcal{F}_{t}\right\}_{t \geq 0}$-adapted processes $X(\cdot)$ such that $|X(\cdot)|_{L^{2}(\Omega ; H)} \in C([0, T])$. All of the above spaces are endowed with the canonical norm.

Denote by $\nu(x)$ the unit outward normal vector of $G$ at $x \in \Gamma$. Let $x_{0} \in\left(\mathbb{R}^{n} \backslash \bar{G}\right)$. In what follows, we choose

$$
\Gamma_{0}=\left\{x \in \Gamma:\left(x-x_{0}\right) \cdot \nu(x)>0\right\} .
$$

The main purpose of this paper is to study the exact controllability of the following controlled linear stochastic Schrödinger equation

$$
\begin{cases}i d y+\Delta y d t=\left(a_{1} \cdot \nabla y+a_{2} y+f\right) d t+\left(a_{3} y+g\right) d B(t) & \text { in } Q, \\ y=0 & \text { on } \Sigma \backslash \Sigma_{0}, \\ y=u & \text { on } \Sigma_{0}, \\ y(0)=y_{0} & \text { in } G .\end{cases}
$$

Here, the initial state $y_{0} \in L^{2}\left(\Omega, \mathcal{F}_{0}, P ; H^{-1}(G)\right)$, the control

$$
u \in L_{\mathcal{F}}^{2}\left(0, T ; L^{2}\left(\Gamma_{0}\right)\right), \quad g \in L^{2}\left(0, T ; H^{-1}(G)\right),
$$

the nonhomogeneous term $f \in L_{\mathcal{F}}^{2}\left(0, T ; L^{2}(G)\right)$ and the coefficients $a_{i}(i=1,2,3)$ satisfy

$$
\left\{\begin{array}{l}
i a_{1} \in L_{\mathcal{F}}^{\infty}\left(0, T ; W^{2, \infty}\left(G ; \mathbb{R}^{n}\right) \cap W_{0}^{1, \infty}\left(G ; \mathbb{R}^{n}\right)\right) \\
a_{2} \in L_{\mathcal{F}}^{\infty}\left(0, T ; W^{1, \infty}(G)\right) \\
a_{3} \in L_{\mathcal{F}}^{\infty}\left(0, T ; W^{1, \infty}(G)\right)
\end{array}\right.
$$

System (1.2) has a nonhomogeneous boundary condition. As the deterministic nonhomogeneous boundary problem, the solution to (1.2) is understood in the transposition sense. Hence, we first introduce the following backward stochastic Schrödinger equation

$$
\begin{cases}i d z+\Delta z d t=\left(b_{1} \cdot \nabla z+b_{2} z+b_{3} Z\right) d t+Z d B(t) & \text { in }(0, \tau) \times G, \\ z=0 & \text { on }(0, \tau) \times \Gamma, \\ z(\tau)=z_{\tau} & \text { in } G,\end{cases}
$$

where $z_{T} \in L_{\mathcal{F}_{T}}^{2}\left(\Omega ; H_{0}^{1}(G)\right)$, the coefficients $b_{i}(i=1,2,3)$ satisfy

$$
\left\{\begin{array}{l}
i b_{1} \in L_{\mathcal{F}}^{\infty}\left(0, T ; W_{0}^{1, \infty}\left(G ; \mathbb{R}^{n}\right)\right) \\
b_{2} \in L_{\mathcal{F}}^{\infty}\left(0, T ; W^{1, \infty}(G)\right) \\
b_{3} \in L_{\mathcal{F}}^{\infty}\left(0, T ; W^{1, \infty}(G)\right)
\end{array}\right.
$$

For the convenience of the reader, we recall the definition of the solution to (1.4) first.

Definition 1.1 A solution to the equation (1.4) is a pair of stochastic processes

$$
(z, Z) \in L_{\mathcal{F}}^{\infty}\left(0, T ; H_{0}^{1}(G)\right) \times L_{\mathcal{F}}^{2}\left(0, T ; H_{0}^{1}(G)\right)
$$


such that for every $\psi \in C_{0}^{\infty}(G)$ and a.e. $(t, \omega) \in[0, \tau] \times \Omega$, it holds that

$$
\begin{aligned}
& \int_{G} i z_{\tau}(x) \psi(x) d x-\int_{G} i z(t) \psi(x) d x-\int_{t}^{\tau} \int_{G} \nabla z(s, x) \cdot \nabla \psi(x) d x d s \\
= & \int_{t}^{\tau} \int_{G}\left[b_{1}(s, x) \nabla z(s, x)+b_{2}(s, x) z(s, x)+b_{3}(s, x) Z(s, x)\right] \psi(x) d x d s \\
& +\int_{t}^{\tau} \int_{G} Z(s, x) \psi(x) d x d B(s) .
\end{aligned}
$$

Let us recall the well-posedness result of (1.4) (see [1, 19] for the proof).

Lemma 1.1 For any $z_{\tau} \in L^{2}\left(\Omega, \mathcal{F}_{\tau}, P ; H_{0}^{1}(G)\right)$, the equation (1.4) admits a unique solution $(z, Z)$. Moreover, $(z, Z)$ satisfies that

$$
|z|_{L_{\mathcal{F}}^{\infty}\left(0, \tau ; H_{0}^{1}(G)\right)}+|Z|_{L_{\mathcal{F}}^{2}\left(0, \tau ; H_{0}^{1}(G)\right)} \leq e^{C r_{1}}\left|z_{\tau}\right|_{L^{2}\left(\Omega, \mathcal{F}_{\tau}, P ; H_{0}^{1}(G)\right)},
$$

where

$$
r_{1} \triangleq\left|b_{1}\right|_{L_{\mathcal{F}}^{\infty}\left(0, T ; W^{1, \infty}\left(G ; \mathbb{R}^{n}\right)\right)}^{2}+\sum_{i=2}^{3}\left|b_{i}\right|_{L_{\mathcal{F}}^{\infty}\left(0, T ; W^{1, \infty}(G)\right)}^{2}+1 .
$$

Here and in the sequel, we will use $C$ to denote a generic positive constant depends on $T$, $G, \Gamma_{0}$ (unless otherwise stated), which may vary from line to line.

Further, we need the following result for the hidden regularity of the solution to (1.4).

Proposition 1.1 Let $z$ be a solution to (1.4), then $\left.\frac{\partial z}{\partial \nu}\right|_{\Gamma} \in L_{\mathcal{F}}^{2}\left(0, \tau ; L^{2}(\Gamma)\right)$. Further, we have the following estimate

$$
\left|\frac{\partial z}{\partial \nu}\right|_{L_{\mathcal{F}}^{2}\left(0, \tau ; L^{2}(\Gamma)\right)} \leq e^{C r_{1}}\left|z_{\tau}\right|_{L_{\mathcal{F}_{\tau}}^{2}\left(\Omega ; H_{0}^{1}(G)\right)}
$$

Remark 1.1 Proposition 1.1 shows that, solutions of (1.4) enjoy a higher regularity on the boundary than the one provided by the classical trace theorem of Sobolev spaces. Such kind of result is called hidden regularity of the solution. There are a great many studies in this topic for deterministic partial differential equations in the literature(see [12, 15] for example).

Now we can give the definition of the solution to (1.2).

Definition $1.2 A$ solution to the system (1.2) is a process $y \in C_{\mathcal{F}}\left([0, T] ; L^{2}\left(\Omega ; H^{-1}(G)\right)\right)$ such that for every $\tau \in[0, T]$ and every $z_{\tau} \in L_{\mathcal{F}_{\tau}}^{2}\left(\Omega ; H_{0}^{1}(G)\right)$ it holds that

$$
\begin{aligned}
& \mathbb{E} \int_{G} y(\tau, x) z_{\tau}(x) d x-\mathbb{E} \int_{G} y_{0}(x) z(0) d x \\
= & \mathbb{E} \int_{0}^{\tau} \int_{\Gamma_{0}} \frac{\partial z}{\partial \nu} u d x d s+\mathbb{E} \int_{0}^{\tau} \int_{G} f z d x d t+\mathbb{E} \int_{0}^{\tau} \int_{G} g Z d x d t .
\end{aligned}
$$

Here $(z, Z)$ solves (1.4) with

$$
b_{1}=-a_{1}, \quad b_{2}=-\operatorname{div}\left(a_{1}\right)+a_{2}, b_{3}=-a_{3} .
$$


Remark 1.2 The solution to (1.2) is defined in the transposition sense. It is well studied that such kind of solutions for deterministic nonhomogeneous boundary value problems in the literature(see [15, 16] for example). On the other hand, the stochastic counterpart is almost open. We only consider a very special problem in this topic. The study of general stochastic nonhomogeneous boundary value problems are very interesting but difficult problems, which is far beyond the scope of this paper.

We have the following well-posedness result for (1.2).

Proposition 1.2 For each $y_{0} \in L_{\mathcal{F}_{0}}^{2}\left(\Omega ; H^{-1}(G)\right)$, the system (1.2) admits a unique solution $y$. Further, for every $y_{0} \in L_{\mathcal{F}_{0}}^{2}\left(\Omega ; H^{-1}(G)\right)$, it holds that

$$
\begin{aligned}
& |y|_{C_{\mathcal{F}}\left([0, T] ; L^{2}\left(\Omega ; H^{-1}(G)\right)\right)} \\
\leq & e^{C r_{1}}\left(\mathbb{E}\left|y_{0}\right|_{H^{-1}(G)}+|f|_{L_{\mathcal{F}}^{2}\left(0, T ; L^{2}(G)\right)}+|u|_{L_{\mathcal{F}}^{2}\left(0, T ; L^{2}\left(\Gamma_{0}\right)\right)}+|g|_{L_{\mathcal{F}}^{2}\left(0, T ; H^{-1}(G)\right)}\right) .
\end{aligned}
$$

Here

$$
r_{2}=\left|a_{1}\right|_{L_{\mathcal{F}}^{\infty}\left(0, T ; W_{0}^{1, \infty}\left(G ; \mathbb{R}^{n}\right)\right)}^{2}+\left|a_{2}\right|_{L_{\mathcal{F}}^{\infty}\left(0, T ; W^{1, \infty}(G)\right)}^{2}+\left|a_{3}\right|_{L_{\mathcal{F}}^{\infty}\left(0, T ; W^{1, \infty}(G)\right)}^{2}+1 .
$$

Now we can give the definition of the exact controllability of (1.2).

Definition 1.3 System (1.2) is said to be exactly controllable at time $T$ if for every initial state $y_{0} \in L_{\mathcal{F}_{0}}^{2}\left(\Omega ; H^{-1}(G)\right)$ and every $y_{1} \in L_{\mathcal{F}_{T}}^{2}\left(\Omega ; H^{-1}(G)\right)$, one can find a pair of controls $(u, g) \in L_{\mathcal{F}}^{2}\left(0, T ; L^{2}\left(\Gamma_{0}\right)\right) \times L_{\mathcal{F}}^{2}\left(0, T ; H^{-1}(G)\right)$ such that the solution $y$ of the system (1.2) satisfies that $y(T)=y_{1}$ in $L_{\mathcal{F}_{T}}^{2}\left(\Omega ; H^{-1}(G)\right)$.

As the deterministic case, the exact controllability of (1.2) can be reduced to an observability estimate of its dual system, that is, the equation (1.4) with $\tau=T$ in our case. For the latter one, we will prove the following result.

Theorem 1.1 All solutions of the equation (1.4) satisfy that

$$
\left|z_{T}\right|_{L_{\mathcal{F}_{T}}^{2}\left(\Omega ; H_{0}^{1}(G)\right)} \leq e^{C r_{1}}\left(|z|_{L_{\mathcal{F}}^{2}\left(0, T ; L^{2}\left(\Gamma_{0}\right)\right)}+|Z|_{L_{\mathcal{F}}^{2}\left(0, T ; H_{0}^{1}(G)\right)}\right) .
$$

By means of Theorem 1.1, we can obtain the following the exact controllability result of the system (1.2).

Theorem 1.2 System (1.2) is exactly controllable at any time $T>0$.

Further, we also have the following result about the lack of exact controllability result for (1.2) if the control $u$ in the drift term is zero.

Theorem 1.3 If $u \equiv 0$ in (1.2), then (1.2) is not exactly controllable at any time $T$.

Remark 1.3 According to the lack of exact controllability for linear stochastic ordinary differential equations in [21], it is clear that the internal control $g$ is necessary for the exact controllability of the system (1.2). From Theorem 1.3, we know that $u$ is also necessary. Hence, we have that one should utilize both $u$ and $g$ to get the exact controllability of (1.2). 
There exist many approaches and results addressing the controllability problem for determinisitc Schrödinger equations. 24 is a nice survey for the works in this respect before 2002. For the works after 2002, we refer the readers to [3, 4, 5, 6, 8, 22] and the rich references therein. However, people know very little about the stochastic counterpart. To our best knowledge, there is no published result for this problem.

Generally speaking, there are four main methods for the exact controllability of deterministic Schrödinger equations.

The first one is the classical Rellich-type multiplier approach ([20]). It can be applied to treat Schrödinger equations with no lower order terms or lower order terms with constant or small coefficients. On the other hand, it seems that it cannot be used to solve our problem since we do not assume that the coefficients of lower order terms are constant or small.

The second one is the microlocal analysis approach ([14]). This method was first introduced in [2] for obtaining the exact controllability of wave equations. It is useful to solve the exact controllability problem for many kinds of partial differential equations such as wave equations, Schrödinger equations and plate equations. However, it seems that there are lots of obstacles needing to be surmounted if one utilize it to study the stochastic control problem(see remarks in Section 6 for more details).

The third one is based on the Ingham type inequality([11]). This method works well for Schrödinger equations involved in some special domains, i.e., intervals, rectangles and balls. However, it seems that it is very hard to applied to equations in general domains.

The last one is the global Carleman estimate([13]). It can be regarded as a more developed version of the classical multiplier method. With respect to the method of multipliers, the Carleman approach has the advantage of being more flexible and allowing to address variable coefficients, with respect to the microlocal one, that it requires less regularity on coefficients and domain, and respect to the method based on Ingham type inequality, that is requires less restrictions to the domain. Further, it is robust with respect to the lower order terms and can be used to get explicit bounds on the observability constant/control cost in terms of the potentials entering in it. This is particularly important when dealing with nonlinear problems by combing linearization and fixed point techniques.

Similar to the deterministic setting, we use a stochastic version of the global Carleman estimate to derive Theorem 1.1. For this, we borrow some idea from the proof of the observability estimate for deterministic Schrödinger equations (see [13] for example). However, the stochastic setting will produce some more troubles. We cannot simply mimic the method in [13. to solve our problem. To handle these troubles, we choose a different weight function for the Carleman estimate.

\section{Some preliminaries}

In this section, we give some preliminary results.

First, we prove the well-posedness of (1.2) in the sense of Definition 1.2,

Proof of Proposition 1.2: Uniqueness of the solution. Suppose there are $y_{1}(\cdot)$ and $y_{2}(\cdot)$ belong to $C_{\mathcal{F}}\left([0, T] ; L^{2}\left(\Omega ; H^{-1}(G)\right)\right)$ such that (1.9) holds. Then, we see

$$
\mathbb{E} \int_{G} y_{1}(\tau, x) z_{\tau}(x) d x=\mathbb{E} \int_{G} y_{2}(\tau, x) z_{\tau}(x) d x \quad \text { for all } z_{\tau} \in L_{\mathcal{F}_{\tau}}^{2}\left(\Omega ; H_{0}^{1}(G)\right) .
$$


This concludes that $y_{1}=y_{2}$.

Existence of the solution. Let us define a linear functional $F$ on $L_{\mathcal{F}_{\tau}}^{2}\left(\Omega ; H_{0}^{1}(G)\right)$ as

$$
F\left(z_{\tau}\right)=\left(y_{0}, z(0)\right)_{H^{-1}(G), H_{0}^{1}(G)}+\mathbb{E} \int_{0}^{\tau} \int_{\Gamma_{0}} \frac{\partial z}{\partial \nu} u d x d s+\mathbb{E} \int_{0}^{\tau} \int_{G} f z d x d t+\mathbb{E} \int_{0}^{\tau} \int_{G} g Z d x d t
$$

It is easy to show that $F$ is a bounded linear functional on $L_{\mathcal{F}_{\tau}}^{2}\left(\Omega ; H_{0}^{1}(G)\right)$. By Riesz Representation Theorem, we know there exists a $y_{\tau} \in L_{\mathcal{F}_{\tau}}^{2}\left(\Omega ; H^{-1}(G)\right)$ such that

$$
F\left(z_{\tau}\right)=\mathbb{E}\left(y_{\tau}, z_{\tau}\right)_{H^{-1}(G), H_{0}^{1}(G)}
$$

Define a process $y(\cdot)$ by $y(\tau)=y_{\tau}$. Now we prove that $y(\cdot) \in C_{\mathcal{F}}\left([0, T] ; L^{2}\left(\Omega ; H^{-1}(G)\right)\right)$.

Let $\xi \in L_{\mathcal{F}_{T}}^{2}\left(\Omega ; H_{0}^{1}(G)\right)$. Consider the following forward random Schrödinger equation

$$
\begin{cases}i d \tilde{z}+\Delta \tilde{z} d t=\left(-a_{1} \cdot \nabla \tilde{z}-\operatorname{div} a_{1} \tilde{z}+a_{2} \tilde{z}\right) d t & \text { in }(\tau, \tau+\delta) \times G, \\ \tilde{z}=0 & \text { on }(\tau, \tau+\delta) \times \Gamma, \\ \tilde{z}(\tau)=\mathbb{E}\left(\xi \mid \mathcal{F}_{\tau}\right) & \text { in } G,\end{cases}
$$

It is easy to see that

$$
\lim _{\delta \rightarrow 0^{+}} \mathbb{E}|\tilde{z}(\tau+\delta)-\tilde{z}(\tau)|_{H_{0}^{1}(G)}^{2}=0
$$

Further, since $\left\{\mathcal{F}_{t}\right\}_{t \geq 0}$ is the natural filtration of $\{B(t)\}_{t \geq 0}$, we have

$$
\lim _{\delta \rightarrow 0^{+}} \mathbb{E}\left|\mathbb{E}\left(\xi \mid \mathcal{F}_{\tau+\delta}\right)-\mathbb{E}\left(\xi \mid \mathcal{F}_{\tau}\right)\right|_{H_{0}^{1}(G)}^{2}=0
$$

From (2.2) and (2.3), we see

$$
\lim _{\delta \rightarrow 0^{+}} \mathbb{E}\left|\tilde{z}(\tau+\delta)-\mathbb{E}\left(z \mid \mathcal{F}_{\tau+\delta}\right)\right|_{H_{0}^{1}(G)}^{2}=0
$$

Let $\left(z_{1}(\cdot), Z_{1}(\cdot)\right)$ and $\left(z_{2}(\cdot), Z_{2}(\cdot)\right)$ be the solution to (1.4) with final data $\mathbb{E}\left(\xi \mid \mathcal{F}_{\tau+\delta}\right)$ and $\tilde{z}(\tau+\delta)$, respectively. Then, from Lemma 1.1 and Proposition 1.1, we know

$$
\left\{\begin{array}{l}
\lim _{\delta \rightarrow 0^{+}}\left|z_{1}-z_{2}\right|_{L_{\mathcal{F}}^{2}\left(0, \tau ; L^{2}(G)\right)}=0, \\
\lim _{\delta \rightarrow 0^{+}}\left|Z_{1}-Z_{2}\right|_{L_{\mathcal{F}}^{2}\left(0, \tau ; L^{2}\left(\Gamma_{0}\right)\right)}=0 \\
\lim _{\delta \rightarrow 0^{+}}\left|\frac{\partial z_{1}}{\partial \nu}-\frac{\partial z_{2}}{\partial \nu}\right|_{L_{\mathcal{F}}^{2}\left(0, \tau ; L^{2}\left(\Gamma_{0}\right)\right)}=0 \\
\lim _{\delta \rightarrow 0^{+}}\left|z_{1}(0)-z_{2}(0)\right|_{H_{0}^{1}(G)}=0 .
\end{array}\right.
$$

Denote by $\left(z_{3}, Z_{3}\right)$ the solution to (1.4) with the final datum $z_{3}(\tau)=\mathbb{E}\left(\xi \mid \mathcal{F}_{\tau}\right)$. From the uniqueness of the solution to (2.1) and (1.4), we find that

$$
z_{3}=z_{2} \text { in }[0, \tau] \times G \text { and } Z_{3}=Z_{2} \text { in }[0, \tau] \times G \text {. }
$$


From the definition of the solution to (1.2), we have

$$
\begin{aligned}
& \mathbb{E}(y(\tau+\delta)-y(\tau), \xi)_{H^{-1}(G), H_{0}^{1}(G)} \\
= & \mathbb{E}(y(\tau+\delta), \xi)_{H^{-1}(G), H_{0}^{1}(G)}-\mathbb{E}(y(\tau), \xi)_{H^{-1}(G), H_{0}^{1}(G)} \\
= & \mathbb{E}\left(y(\tau+\delta), \mathbb{E}\left(\xi \mid \mathcal{F}_{\tau+\delta}\right)\right)_{H^{-1}(G), H_{0}^{1}(G)}-\mathbb{E}\left(y(\tau), \mathbb{E}\left(\xi \mid \mathcal{F}_{\tau}\right)\right)_{H^{-1}(G), H_{0}^{1}(G)} \\
= & \mathbb{E}\left(y_{0}, z_{1}(0)-z_{3}(0)\right)_{H^{-1}(G), H_{0}^{1}(G)}+\mathbb{E} \int_{0}^{\tau} \int_{\Gamma_{0}}\left(\frac{\partial z_{1}}{\partial \nu}-\frac{\partial z_{3}}{\partial \nu}\right) u d \Gamma d s \\
& +\mathbb{E} \int_{0}^{\tau} \int_{G} f\left(z_{1}-z_{3}\right) d x d t+\mathbb{E} \int_{0}^{\tau} \int_{G} g\left(Z_{1}-Z_{3}\right) d x d t+\mathbb{E} \int_{\tau}^{\tau+\delta} \int_{\Gamma_{0}} \frac{\partial z_{1}}{\partial \nu} u d \Gamma d s \\
& +\mathbb{E} \int_{\tau}^{\tau+\delta} \int_{G} f z_{1} d x d t+\mathbb{E} \int_{\tau}^{\tau+\delta} \int_{G} g Z_{1} d x d t .
\end{aligned}
$$

This, together with (2.5) and (2.6), implies that

$$
\lim _{\delta \rightarrow 0^{+}} \mathbb{E}(y(\tau+\delta)-y(\tau), \xi)_{H^{-1}(G), H_{0}^{1}(G)}=0, \quad \text { for any } \xi \in L_{\mathcal{F}_{T}}^{2}\left(\Omega ; H_{0}^{1}(G)\right) .
$$

Similarly, we can show that

$$
\lim _{\delta \rightarrow 0^{-}} \mathbb{E}(y(\tau+\delta)-y(\tau), \xi)_{H^{-1}(G), H_{0}^{1}(G)}=0, \quad \text { for any } \xi \in L_{\mathcal{F}_{T}}^{2}\left(\Omega ; H_{0}^{1}(G)\right) .
$$

Hence, we see $y(\cdot) \in C_{\mathcal{F}}\left([0, T] ; L^{2}\left(\Omega ; H^{-1}(G)\right)\right)$.

Next, for the sake of completeness, we give an energy estimate for the equation (1.4).

Proposition 2.1 For all $z$ which solve the equation (1.4), it holds that

$$
\mathbb{E}|z(t)|_{H_{0}^{1}(G)}^{2} \leq e^{C r_{1}}\left(\mathbb{E}|z(s)|_{H_{0}^{1}(G)}^{2}+|Z|_{L_{\mathcal{F}}^{2}\left(0, T ; H_{0}^{1}(G)\right)}^{2}\right),
$$

for any $0 \leq s \leq t \leq \tau$.

Proof: By direct computation, we have

$$
\begin{aligned}
& \quad \mathbb{E}|z(t)|_{L^{2}(G)}^{2}-\mathbb{E}|z(s)|_{L^{2}(G)}^{2} \\
& =\mathbb{E} \int_{s}^{t} \int_{G}(z d \bar{z}+\bar{z} d z+d z d \bar{z}) d x \\
& =\mathbb{E} \int_{s}^{t} \int_{G}\left\{-i z\left(\Delta \bar{z}+b_{1} \cdot \nabla \bar{z}-b_{2} \bar{z}-b_{3} \bar{Z}\right)\right. \\
& \left.\quad+i \bar{z}\left(\Delta z-b_{1} \cdot \nabla z-b_{2} z-b_{3} Z\right)+Z \bar{Z}\right\} d x d \sigma \\
& =\mathbb{E} \int_{s}^{t} \int_{G}\left\{-i\left[\operatorname{div}(z \nabla \bar{z})-|\nabla z|^{2}+\operatorname{div}\left(|z|^{2} b_{1}\right)-\operatorname{div}\left(b_{1}\right)|z|^{2}-b_{2}|z|^{2}-b_{3} z \bar{Z}\right]\right. \\
& \left.\quad+i\left[\operatorname{div}(\bar{z} \nabla z)-|\nabla z|^{2}-b_{2}|z|^{2}-b_{3} \bar{z} Z\right]+|Z|^{2}\right\} d x d \sigma \\
& \leq \mathbb{E} \int_{s}^{t} 2\left[\left(\left|b_{1}\right|_{W^{1, \infty}\left(G ; \mathbb{R}^{n}\right)}+\left|b_{3}\right|_{L^{\infty}(G)}+1\right)|z|_{L^{2}(G)}^{2}+|Z|_{L^{2}(G)}^{2}\right] d x d \sigma
\end{aligned}
$$


and

$$
\begin{aligned}
& \mathbb{E}|\nabla z(t)|_{L^{2}(G)}^{2}-\mathbb{E}|\nabla z(s)|_{L^{2}(G)}^{2} \\
= & \mathbb{E} \int_{s}^{t} \int_{G}(\nabla z d \nabla \bar{z}+\nabla \bar{z} d \nabla z+d \nabla z d \nabla \bar{z}) d x \\
= & \mathbb{E} \int_{s}^{t} \int_{G}\{\operatorname{div}(\nabla z d \bar{z})-\Delta z d \bar{z}+\operatorname{div}(\nabla \bar{z} d z)-\Delta \bar{z} d z+d \nabla z d \nabla \bar{z}\} d x \\
= & \mathbb{E} \int_{s}^{t} \int_{G}\left\{\Delta z\left[i\left(\Delta \bar{z}+b_{1} \cdot \nabla \bar{z}-b_{2} \bar{z}-b_{3} \bar{Z}\right)\right]\right. \\
\leq & 2 \mathbb{E} \int_{t}^{s}\left\{\left(\left|b_{1}\right|_{W^{1, \infty}\left(G ; \mathbb{R}^{n}\right)}^{2}+\left|b_{3}\right|_{W^{1, \infty}(G)}^{2}+1\right)|\nabla z|_{L^{2}(G)}^{2}\left[i\left(\Delta z-b_{1} \cdot \nabla z-b_{2} z-b_{3} Z\right)\right]+|\nabla Z|^{2}\right\} d x d \sigma \\
& \left.+\left(\left|b_{2}\right|_{W^{1, \infty}(G)}^{2}+\left|b_{3}\right|_{W^{1, \infty}(G)}^{2}+1\right)|z|_{L^{2}(G)}^{2}+|Z|_{H_{0}^{1}(G)}^{2}\right\} d \sigma .
\end{aligned}
$$

From (2.8) and (2.9), we get

$$
\begin{aligned}
& \mathbb{E}|z(t)|_{H_{0}^{1}(G)}^{2}-\mathbb{E}|z(s)|_{H_{0}^{1}(G)}^{2} \\
\leq & 2\left(r_{1}+1\right) \mathbb{E} \int_{t}^{s}|z(\sigma)|_{H_{0}^{1}(G)}^{2} d \sigma+\mathbb{E} \int_{t}^{s}|Z(\sigma)|_{H_{0}^{1}(G)}^{2} d \sigma .
\end{aligned}
$$

From (2.10), and thanks to Gronwall's inequality, we arrive at

$$
\mathbb{E}|y(t)|_{H_{0}^{1}(G)}^{2} \leq e^{2\left(r_{1}+1\right)}\left\{\mathbb{E}|y(s)|_{H_{0}^{1}(G)}^{2}+\mathbb{E} \int_{0}^{\tau}|Z|_{H_{0}^{1}(G)}^{2} d \sigma\right\},
$$

which implies the inequality (2.7) immediately.

Remark 2.1 The proof of this proposition is almost standard. Indeed, if we regard $z$ as a solution to a forward stochastic Schrödinger equation with a nonhomogeneous term $Z$, then it is a standard energy estimate for such kind of equation.

Next, we give a proof of Proposition 1.1. For this, we first recall a pointwise identity. For simplicity, in what follows, we adopt the notation $z_{i} \equiv z_{i}(x) \triangleq \frac{\partial z(x)}{\partial x_{i}}$, where $x_{i}$ is the $i$-th coordinate of a generic point $x=\left(x_{1}, \cdots, x_{n}\right)$ in $\mathbb{R}^{n}$. In a similar manner, we use the notation $y_{i}, v_{i}$, etc., for the partial derivatives of $y$ and $v$ with respect to $x_{i}$. Let us recall the following identity.

Lemma 2.1 [18, Proposition 2.3] Let $\mu=\mu(x)=\left(\mu^{1}, \cdots, \mu^{n}\right): \mathbb{R}^{n} \rightarrow \mathbb{R}^{n}$ be a vector field of class $C^{1}$ and $z$ an $H_{\text {loc }}^{2}\left(\mathbb{R}^{n}\right)$-valued $\left\{\mathcal{F}_{t}\right\}_{t \geq 0}$-adapted process. Then for a.e. $x \in \mathbb{R}^{n}$ and P-a.s. $\omega \in \Omega$, it holds that

$$
\begin{aligned}
& \mu \cdot \nabla \bar{z}(i d z+\Delta z d t)+\mu \cdot \nabla z(-i d \bar{z}+\Delta \bar{z} d t) \\
= & \nabla \cdot\left[(\mu \cdot \nabla \bar{z}) \nabla z+(\mu \cdot \nabla z) \nabla \bar{z}-i(z d \bar{z}) \mu-|\nabla z|^{2} \mu\right] d t+d(i \mu \cdot \nabla \bar{z} z) \\
& -2 \sum_{j, k=1}^{n} \mu_{j}^{k} z_{j} \bar{z}_{k} d t+(\nabla \cdot \mu)|\nabla z|^{2} d t+i(\nabla \cdot \mu) z d \bar{z}-i(\mu \cdot \nabla d \bar{z}) d z .
\end{aligned}
$$


By virtue of Lemma 2.1, the proof of Proposition 1.1 is standard. We only give a sketch here.

Sketch of the Proof of Proposition 1.1: Since $\Gamma$ is $C^{2}$, one can find a vector field $\mu_{0}=$ $\left(\mu_{0}^{1}, \cdots, \mu_{0}^{n}\right) \in C^{1}\left(\bar{G} ; \mathbb{R}^{n}\right)$ such that $\mu_{0}=\nu$ on $\Gamma$ (see [10, page 18] for the construction of $\left.\mu_{0}\right)$. Letting $\mu=\mu_{0}$ and $z=y$ in Lemma 2.1, integrating it in $Q$ and taking the expectation, by means of Proposition 2.1, with similar computation in [23], Proposition 1.1 can be obtained immediately.

Next, we recall an identity in the spirit of (2.12) but much more complex, which will play an important role in establishing the Carleman estimate for (1.4).

Let $\beta(t, x) \in C^{2}\left(\mathbb{R}^{1+n} ; \mathbb{R}\right)$, and let $b^{j k}(t, x) \in C^{1,2}\left(\mathbb{R}^{1+n} ; \mathbb{R}\right)$ satisfy

$$
b^{j k}=b^{k j}, \quad j, k=1,2, \cdots, n .
$$

Let us define a (formal) second order stochastic partial differential operator $\mathcal{P}$ as

$$
\mathcal{P} z \triangleq i \beta(t, x) d w+\sum_{j, k=1}^{n}\left(b^{j k}(t, x) w_{j}\right)_{k} d t, \quad i=\sqrt{-1} .
$$

We have the following equality concerning $\mathcal{P}$ :

Lemma $2.2\left[18\right.$, Theorem 3.1] Let $\ell, \Psi \in C^{2}\left(\mathbb{R}^{1+n} ; \mathbb{R}\right)$ and $\theta=e^{\ell}$. Assume that $w$ is an $H_{\text {loc }}^{2}\left(\mathbb{R}^{n}, \mathbb{C}\right)$-valued $\left\{\mathcal{F}_{t}\right\}_{t \geq 0}$-adapted process. Put $v=\theta w$. Then for a.e. $x \in \mathbb{R}^{n}$ and P-a.s. $\omega \in \Omega$, it holds that

$$
\begin{aligned}
& \theta\left(\mathcal{P} w \overline{I_{1}}+\overline{\mathcal{P} w} I_{1}\right)+d M+\operatorname{div} V \\
& =2\left|I_{1}\right|^{2} d t+\sum_{j, k=1}^{n} c^{j k}\left(v_{k} \bar{v}_{j}+\bar{v}_{k} v_{j}\right) d t+D|v|^{2} d t \\
& +i \sum_{j, k=1}^{n}\left[\left(\beta b^{j k} \ell_{j}\right)_{t}+b^{j k}\left(\beta \ell_{t}\right)_{j}\right]\left(\bar{v}_{k} v-v_{k} \bar{v}\right) d t \\
& +i\left[\beta \Psi+\sum_{j, k=1}^{n}\left(\beta b^{j k} \ell_{j}\right)_{k}\right](\bar{v} d v-v d \bar{v}) \\
& +\left(\beta^{2} \ell_{t}\right) d v d \bar{v}+i \sum_{j, k=1}^{n} \beta b^{j k} \ell_{j}\left(d v d \bar{v}_{k}-d v_{k} d \bar{v}\right)
\end{aligned}
$$

where

$$
\left\{\begin{array}{l}
I_{1} \triangleq-i \beta \ell_{t} v-2 \sum_{j, k=1}^{n} b^{j k} \ell_{j} v_{k}+\Psi v \\
A \triangleq \sum_{j, k=1}^{n} b^{j k} \ell_{j} \ell_{k}-\sum_{j, k=1}^{n}\left(b^{j k} \ell_{j}\right)_{k}-\Psi
\end{array}\right.
$$




$$
\left\{\begin{array}{l}
M \triangleq \beta^{2} \ell_{t}|v|^{2}+i \beta \sum_{j, k=1}^{n} b^{j k} \ell_{j}\left(\bar{v}_{k} v-v_{k} \bar{v}\right), \\
V \triangleq\left[V^{1}, \cdots, V^{k}, \cdots, V^{n}\right], \\
V^{k} \triangleq-i \beta \sum_{j=1}^{n}\left[b^{j k} \ell_{j}(v d \bar{v}-\bar{v} d v)+b^{j k} \ell_{t}\left(v_{j} \bar{v}-\bar{v}_{j} v\right) d t\right] \\
\quad-\Psi \sum_{j=1}^{n} b^{j k}\left(v_{j} \bar{v}+\bar{v}_{j} v\right) d t+\sum_{j=1}^{n} b^{j k}\left(2 A \ell_{j}+\Psi_{j}\right)|v|^{2} d t \\
\quad+\sum_{j, j^{\prime}, k^{\prime}=1}^{n}\left(2 b^{j k^{\prime}} b^{j^{\prime} k}-b^{j k} b^{j^{\prime} k^{\prime}}\right) \ell_{j}\left(v_{j^{\prime}} \bar{v}_{k^{\prime}}+\bar{v}_{j^{\prime}} v_{k^{\prime}}\right) d t,
\end{array}\right.
$$

and

$$
\left\{\begin{array}{l}
c^{j k} \triangleq \sum_{j^{\prime}, k^{\prime}=1}^{n}\left[2\left(b^{j^{\prime} k} \ell_{j^{\prime}}\right)_{k^{\prime}} b^{j k^{\prime}}-\left(b^{j k} b^{j^{\prime} k^{\prime}} \ell_{j^{\prime}}\right)_{k^{\prime}}\right]-b^{j k} \Psi, \\
D \triangleq\left(\beta^{2} \ell_{t}\right)_{t}+\sum_{j, k=1}^{n}\left(b^{j k} \Psi_{k}\right)_{j}+2\left[\sum_{j, k=1}^{n}\left(b^{j k} \ell_{j} A\right)_{k}+A \Psi\right] .
\end{array}\right.
$$

\section{A global Carleman estimate for the equation (1.4)}

In this section, we establish a global Carleman estimate for the solution to (1.4) (see Theorem 3.1 below).

To begin with, let us introduce the weight functions to be used in our Carleman estimate. Let

$$
\psi(x)=\left|x-x_{0}\right|^{2}+\sigma,
$$

where $\sigma$ is a positive constant such that $\psi \geq \frac{5}{6}|\psi|_{L^{\infty}(G)}$. Let $s>0$ and $\lambda>0$. Put

$$
\ell=s \frac{e^{4 \lambda \psi}-e^{5 \lambda|\psi|_{L} \infty(G)}}{t^{2}(T-t)^{2}}, \quad \varphi=\frac{e^{4 \lambda \psi}}{t^{2}(T-t)^{2}} .
$$

We have the following global Carleman inequality.

Theorem 3.1 According to (3.2), there is an $s_{1}>0$ (depending on $r_{1}$ ) and a $\lambda_{1}>0$ such that for each $s \geq s_{1}, \lambda \geq \lambda_{1}$ and for any solution of the equation (1.4), it holds that

$$
\begin{aligned}
& \mathbb{E} \int_{Q} \theta^{2}\left(s^{3} \lambda^{4} \varphi^{3}|z|^{2}+s \lambda \varphi|\nabla z|^{2}\right) d x d t \\
\leq & C\left[\mathbb{E} \int_{Q} \theta^{2}\left(s^{2} \lambda^{2} \varphi^{2}|Z|^{2}+|\nabla Z|^{2}\right) d x d t+\mathbb{E} \int_{0}^{T} \int_{\Gamma_{0}} \theta^{2} s \lambda \varphi\left|\frac{\partial z}{\partial \nu}\right|^{2} d \Gamma d t\right] .
\end{aligned}
$$

Proof of Theorem [3.1] The proof is divided into three steps. 
Step 1. We choose $\beta=1$ and $\left(b^{j k}\right)_{1 \leq j, k \leq n}$ to be the identity matrix. Put

$$
\delta^{j k}= \begin{cases}1, & \text { if } j=k, \\ 0, & \text { if } j \neq k .\end{cases}
$$

Applying Lemma 2.2 to the equation (1.4) with $\theta$ given by (3.2), $w$ replaced by $z$ and $v=\theta z$, we obtain that

$$
\begin{aligned}
& \theta \mathcal{P} z\left(i \beta \ell_{t} \bar{v}-2 \sum_{j, k=1}^{n} b^{j k} \ell_{j} \bar{v}_{k}+\Psi \bar{v}\right)+\theta \overline{\mathcal{P} z}\left(-i \beta \ell_{t} v-2 \sum_{j, k=1}^{n} b^{j k} \ell_{j} v_{k}+\Psi v\right) \\
& \quad+d M+\operatorname{div} V \\
& =2\left|-i \beta \ell_{t} v-2 \sum_{j, k=1}^{n} b^{j k} \ell_{j} v_{k}+\Psi v\right|^{2} d t+\sum_{j, k=1}^{n} c^{j k}\left(v_{k} \bar{v}_{j}+\bar{v}_{k} v_{j}\right) d t+D|v|^{2} d t \\
& +2 i \sum_{j=1}^{n}\left(\ell_{j t}+\ell_{t j}\right)\left(\bar{v}_{j} v-v_{j} \bar{v}\right) d t+i(\Psi+\Delta \ell)(\bar{v} d v-v d \bar{v}) \\
& +\ell_{t} d v d \bar{v}+i \sum_{j=1}^{n} \ell_{j}\left(d \bar{v}_{j} d v-d v_{j} d \bar{v}\right) .
\end{aligned}
$$

Here

$$
\begin{gathered}
M=\beta^{2} \ell_{t}|v|^{2}+i \beta \sum_{j, k=1}^{n} b^{j k} \ell_{j}\left(\bar{v}_{k} v-v_{k} \bar{v}\right) \\
=\ell_{t}|v|^{2}+i \sum_{j=1}^{n} \ell_{j}\left(\bar{v}_{j} v-v_{j} \bar{v}\right) ; \\
A=\sum_{j, k=1}^{n} b^{j k} \ell_{j} \ell_{k}-\sum_{j, k=1}^{n}\left(b^{j k} \ell_{j}\right)_{k}-\Psi \\
=\sum_{j=1}^{n}\left(\ell_{j}^{2}-\ell_{j j}\right)-\Psi ; \\
=\left(\beta^{2} \ell_{t}\right)_{t}+\sum_{j, k=1}^{n}\left(b^{j k} \Psi_{k}\right)_{j}+2\left[\sum_{j, k=1}^{n}\left(b^{j k} \ell_{j} A\right)_{k}+A \Psi\right] \\
\ell_{t t}+\sum_{j=1}^{n} \Psi_{j j}+2 \sum_{j=1}^{n}\left(\ell_{j} A\right)_{j}+2 A \Psi ; \\
=\sum_{j^{\prime}, k^{\prime}=1}^{n}\left[2\left(b^{j^{\prime} k} \ell_{j^{\prime}}\right)_{k^{\prime}} b^{j k^{\prime}}-\left(b^{j k} b^{j^{\prime} k^{\prime}} \ell_{j^{\prime}}\right)_{k^{\prime}} \Psi\right]-b^{j k} \\
=\left[2\left(b^{k k} \ell_{k}\right)_{j} b^{j j}-\sum_{j^{\prime}=1}^{n}\left(b^{j k} b^{j^{\prime} j^{\prime}} \ell_{j^{\prime}}\right)_{j^{\prime}}-b^{j k} \Psi\right] \\
\ell_{j k}-\delta^{j k} \Delta \ell-\delta^{j k} \Psi ;
\end{gathered}
$$


and

$$
\begin{aligned}
V_{k}= & -i \beta \sum_{j=1}^{n}\left[b^{j k} \ell_{j}(v d \bar{v}-\bar{v} d v)+b^{j k} \ell_{t}\left(v_{j} \bar{v}-\bar{v}_{j} v\right) d t\right] \\
& -\Psi \sum_{j=1}^{n} b^{j k}\left(v_{j} \bar{v}+\bar{v}_{j} v\right) d t+\sum_{j=1}^{n} b^{j k}\left(2 A \ell_{j}+\Psi_{j}\right)|v|^{2} d t \\
& +\sum_{j, j^{\prime}, k^{\prime}=1}^{n}\left(2 b^{j k^{\prime}} b^{j^{\prime} k}-b^{j k} b^{j^{\prime} k^{\prime}}\right) \ell_{j}\left(v_{j^{\prime}} \bar{v}_{k^{\prime}}+\bar{v}_{j^{\prime}} v_{k^{\prime}}\right) d t \\
= & -i\left[\ell_{k}(v d \bar{v}-\bar{v} d v)+\ell_{t}\left(v_{j} \bar{v}-\bar{v}_{j} v\right) d t\right]-\Psi\left(v_{k} \bar{v}+\bar{v}_{k} v\right) d t+\left(2 A \ell_{k}+\Psi_{k}\right)|v|^{2} d t \\
& +2 \sum_{j=1}^{n} \ell_{j}\left(\bar{v}_{j} v_{k}+v_{j} \bar{v}_{k}\right) d t-2 \sum_{j^{\prime}=1}^{n} \ell_{k}\left(v_{j} \bar{v}_{j}\right) d t .
\end{aligned}
$$

Step 2. In this step, we estimate the terms in the right-hand side of the equality (3.4) one by one.

First, from the definition of $\ell, \varphi($ see $(3.2))$ and the choice of $\psi($ see $(3.1)$ ), we have

$$
\begin{aligned}
\left|\ell_{t}\right| & =\mid s \frac{2(2 t-T)}{t^{3}(T-t)^{3}}\left(e^{4 \lambda \psi}-e^{\left.5 \lambda|\psi|_{L^{\infty}(G)}\right)} \mid\right. \\
& \leq\left|s \frac{2(2 t-T)}{t^{3}(T-t)^{3}} e^{5 \lambda|\psi|_{L} \infty(G)}\right| \\
& \leq\left|s \frac{C}{t^{3}(T-t)^{3}} e^{5 \lambda \psi}\right| \\
& \leq C s \varphi^{1+\frac{1}{2}}
\end{aligned}
$$

and

$$
\begin{aligned}
\left|\ell_{t t}\right| & =\left|s \frac{20 t^{2}-20 t T+6 T^{2}}{t^{4}(T-t)^{4}}\left(e^{4 \lambda \psi}-e^{5 \lambda|\psi|_{L^{\infty}(G)}}\right)\right| \\
& \leq\left|s \frac{C}{t^{4}(T-t)^{4}} e^{5 \lambda|\psi|_{L^{\infty}(G)}}\right| \\
& \leq\left|s \frac{C}{t^{4}(T-t)^{4}} e^{8 \lambda \psi}\right| \\
& \leq C s \varphi^{2} \leq C s \varphi^{3} .
\end{aligned}
$$

We choose below $\Psi=-\Delta \ell$, then we have that

$$
A=\sum_{j=1}^{n} \ell_{j}^{2}=\sum_{j=1}^{n}\left(4 s \lambda \varphi \psi_{j}\right)^{2}=16 s^{2} \lambda^{2} \varphi^{2}|\nabla \psi|^{2} .
$$


Hence, we find

$$
\begin{aligned}
D & =\ell_{t t}+\sum_{j=1}^{n} \Psi_{j j}+2 \sum_{j=1}^{n}\left(\ell_{j} A\right)_{j}+2 A \Psi \\
& =\ell_{t t}+\Delta(\Delta \ell)+2 \sum_{j=1}^{n}\left(4 s \lambda \varphi \psi_{j} 16 s^{2} \lambda^{2} \varphi^{2}|\nabla \psi|^{2}\right)_{j}-32 s^{2} \lambda^{2} \varphi^{2}|\nabla \psi|^{2} \Delta \ell \\
& =384 s^{3} \lambda^{4} \varphi^{3}|\nabla \psi|^{4}-\lambda^{4} \varphi O(s)-s^{3} \varphi^{3} O\left(\lambda^{3}\right)+\ell_{t t} .
\end{aligned}
$$

Recalling that $x_{0} \in\left(\mathbb{R}^{n} \backslash \bar{G}\right)$, we know that

$$
|\nabla \psi|>0 \text { in } \bar{G} .
$$

From (3.13) and (3.11), we know that there exists a $\lambda_{0}>0$ such that for all $\lambda>\lambda_{0}$, one can find a constant $s_{0}=s_{0}\left(\lambda_{0}\right)$ so that for any $s>s_{0}$, it holds that

$$
D|v|^{2} \geq s^{3} \lambda^{4} \varphi^{3}|\nabla \psi|^{4}|v|^{2} .
$$

Since

$$
\begin{aligned}
c^{j k} & =2 \ell_{j k}-\delta^{j k} \Delta \ell-\delta^{j k} \Psi \\
& =32 s \lambda^{2} \varphi \psi_{j} \psi_{k}+16 s \lambda \varphi \psi_{j k},
\end{aligned}
$$

we see that

$$
\begin{aligned}
& \sum_{j, k=1}^{n} c^{j k}\left(v_{j} \bar{v}_{k}+v_{k} \bar{v}_{j}\right) \\
= & 32 s \lambda^{2} \varphi \sum_{j, k=1}^{n} \psi_{j} \psi_{k}\left(v_{j} \bar{v}_{k}+v_{k} \bar{v}_{j}\right)+16 s \lambda \varphi \sum_{j, k=1}^{n} \psi_{j k}\left(v_{j} \bar{v}_{k}+v_{k} \bar{v}_{j}\right) \\
= & 32 s \lambda^{2} \varphi\left[\sum_{j=1}^{n}\left(\psi_{j} v_{j}\right) \sum_{k=1}^{n}\left(\psi_{k} \bar{v}_{k}\right)+\sum_{k=1}^{n}\left(\psi_{k} v_{k}\right) \sum_{j=1}^{n}\left(\psi_{j} \bar{v}_{j}\right)\right]+32 s \lambda \varphi \sum_{j=1}^{n}\left(v_{j} \bar{v}_{j}+\bar{v}_{j} v_{j}\right) \\
= & 64 s \lambda^{2} \varphi|\nabla \psi \cdot \nabla v|^{2}+64 s \lambda \varphi|\nabla v|^{2} \\
\geq & 64 s \lambda \varphi|\nabla v|^{2} .
\end{aligned}
$$

Now we estimate the other terms in the right-hand side of the equality (3.4). The first one reads

$$
\begin{aligned}
2 i \sum_{j=1}^{n}\left(\ell_{j t}+\ell_{t j}\right)\left(\bar{v}_{j} v-v_{j} \bar{v}\right) & =4 i \sum_{j=1}^{n} s \lambda \psi_{j} \ell_{t}\left(\bar{v}_{j} v-\bar{v} v_{j}\right) \\
& \leq 2 s \varphi|\nabla v|^{2}+2 s \lambda^{2} \varphi^{3}|\nabla \psi|^{2}\left|v^{2}\right| .
\end{aligned}
$$

The second one satisfies

$$
i(\Psi+\Delta \ell)(\bar{v} d v-v d \bar{v})=0 .
$$


For estimating the third and the fourth one, we need to take mean value and get that

$$
\begin{aligned}
\mathbb{E}\left(\ell_{t} d v d \bar{v}\right) & =\mathbb{E}\left[\ell_{t}\left(\theta \ell_{t} z d t+\theta d z\right)\left(\overline{\left.\theta \ell_{t} z d t+\theta d z\right)}\right]=\mathbb{E}\left(\ell_{t} \theta^{2} d z d \bar{z}\right)\right. \\
& \leq 2 s \theta^{2} \varphi^{\frac{3}{2}} \mathbb{E}|Z|^{2} d t
\end{aligned}
$$

Here we utilize the inequality (3.10).

Further,

$$
\begin{aligned}
\mathbb{E}\left(d \bar{v}_{j} d v\right) & =\mathbb{E}\left[\overline{\left(\theta \ell_{t} z d t+\theta d z\right)_{j}}\left(\theta \ell_{t} z d t+\theta d z\right)\right] \\
& =\mathbb{E}\left[\overline{(\theta d z)_{j}}(\theta d z)\right] \\
& =\mathbb{E}\left[\overline{\left(s \lambda \varphi \psi_{j} \theta d z+\theta d z_{j}\right)} \theta d z\right] \\
& =s \lambda \varphi \psi_{j} \theta^{2} \mathbb{E} d \bar{z} d z+\theta^{2} \mathbb{E} d \bar{z}_{j} d z \\
& =s \lambda \varphi \psi_{j} \theta^{2} \mathbb{E}|Z|^{2} d t+\theta^{2} \mathbb{E}\left(\bar{Z}_{j} Z\right) d t
\end{aligned}
$$

Similarly, we can get that

$$
\mathbb{E}\left(d v_{j} d \bar{v}\right)=s \lambda \varphi \psi_{j} \theta^{2} \mathbb{E}|Z|^{2} d t+\theta^{2} \mathbb{E}\left(\bar{Z} Z_{j}\right) d t .
$$

Therefore, the fourth one satisfies that

$$
i \mathbb{E} \sum_{j=1}^{n} \ell_{j}\left(d \bar{v}_{j} d v-d v_{j} d \bar{v}\right)=i s \lambda \varphi \psi_{j}\left[\theta^{2} \mathbb{E}\left(\bar{Z}_{j} Z\right) d t-\theta^{2} \mathbb{E}\left(\bar{Z}_{j} Z\right) d t\right] .
$$

Step 3. Integrating the equality (3.4) in $Q$, taking mean value in both sides, and noting (3.12)-(3.19), we obtain that

$$
\begin{aligned}
& \mathbb{E} \int_{Q}\left(s^{3} \lambda^{4} \varphi^{3}|v|^{2}+s \lambda^{2} \varphi|\nabla v|^{2}\right) d x d t+2 \mathbb{E} \int_{Q}\left|-i \ell_{t} v-2 \sum_{j=1}^{n} \ell_{j} v_{j}+\Psi v\right|^{2} d x d t \\
\leq & \mathbb{E} \int_{Q}\left[\theta \mathcal{P} y\left(i \ell_{t} \bar{v}-2 \sum_{j=1}^{n} \ell_{j} \bar{v}_{j}+\Psi \bar{v}\right)+\theta \overline{\mathcal{P} y}\left(-i \ell_{t} v-2 \sum_{j=1}^{n} \ell_{j} v_{j}+\Psi v\right)\right] d x \\
& +C \mathbb{E} \int_{Q} \theta^{2}\left(s^{2} \lambda^{2} \varphi^{2}|Z|+|\nabla Z|^{2}\right) d x d t+\mathbb{E} \int_{Q} d M d x+\mathbb{E} \int_{Q} \operatorname{div} V d x .
\end{aligned}
$$

Now we analyze the terms in the right-hand side of the inequality (3.20) one by one. The first one reads

$$
\begin{gathered}
\mathbb{E} \int_{Q}\left[\theta \mathcal{P} z\left(i \ell_{t} \bar{v}-2 \sum_{j=1}^{n} \ell_{j} \bar{v}_{j}+\Psi \bar{v}\right)+\theta \overline{\mathcal{P} z}\left(-i \ell_{t} v-2 \sum_{j=1}^{n} \ell_{j} v_{j}+\Psi v\right)\right] d x \\
=\mathbb{E} \int_{Q}\left[\theta\left(b_{1} \cdot \nabla z+b_{2} z+b_{3} Z\right)\left(i \ell_{t} \bar{v}-2 \sum_{j=1}^{n} \ell_{j} \bar{v}_{j}+\Psi \bar{v}\right)\right. \\
\left.+\theta\left(-b_{1} \cdot \nabla \bar{z}+b_{2} \bar{z}+b_{3} \bar{Z}\right)\left(-i \ell_{t} v-2 \sum_{j=1}^{n} \ell_{j} v_{j}+\Psi v\right)\right] d x d t \\
\leq 2 \mathbb{E} \int_{Q}\left\{\theta^{2}\left|b_{1} \cdot \nabla z+b_{2} z+b_{3} Z\right|^{2}+\left|-i \beta \ell_{t} v-2 \sum_{j=1}^{n} \ell_{j} v_{j}+\Psi v\right|^{2}\right\} d x d t .
\end{gathered}
$$


From the choice of $\theta$, we know that $v(0)=v(T)=0$. Hence, we have

$$
\int_{Q} d M d x=0 .
$$

Further, by Stokes' Theorem and noting that $v=z=0$ on $(0, T) \times \Gamma$, we find

$$
\begin{aligned}
\mathbb{E} \int_{Q} \operatorname{div} V d x & =\mathbb{E} \int_{\Sigma} 2 \sum_{k=1}^{n} \sum_{j=1}^{n}\left[\ell_{j}\left(\bar{v}_{j} v_{k}+v_{j} \bar{v}_{k}\right) \nu^{k}-\ell_{k} \nu_{k} v_{j} \bar{v}_{j}\right] d \Sigma \\
& =\mathbb{E} \int_{\Sigma}\left(4 \sum_{j=1}^{n} \ell_{j} \nu_{j}\left|\frac{\partial v}{\partial \nu}\right|^{2}-2 \sum_{k=1}^{n} \ell_{k} \nu_{k}\left|\frac{\partial v}{\partial \nu}\right|^{2}\right) d \Sigma \\
& =\mathbb{E} \int_{\Sigma} 2 \sum_{k=1}^{n} \ell_{k} \nu_{k}\left|\frac{\partial v}{\partial \nu}\right|^{2} d \Sigma \\
& \leq C \mathbb{E} \int_{0}^{T} \int_{\Gamma_{0}} \theta^{2} s \lambda \varphi\left|\frac{\partial z}{\partial \nu}\right|^{2} d \Gamma d t .
\end{aligned}
$$

From (3.20)-3.23), we have

$$
\begin{aligned}
& \mathbb{E} \int_{Q}\left(s^{3} \lambda^{4} \varphi^{3}|v|^{2}+s \lambda \varphi|\nabla v|^{2}\right) d x d t \\
\leq & C \mathbb{E} \int_{Q} \theta^{2}\left|b_{1} \cdot \nabla z+b_{2} z+b_{3} Z\right|^{2} d x d t+C \mathbb{E} \int_{0}^{T} \int_{\Gamma_{0}} \theta^{2} s \lambda \varphi\left|\frac{\partial z}{\partial \nu}\right|^{2} d \Gamma d t \\
& +C \mathbb{E} \int_{Q} \theta^{2}\left(s^{2} \lambda^{2} \varphi^{2}|Z|^{2}+|\nabla Z|^{2}\right) d x d t .
\end{aligned}
$$

Noting that $z_{i}=\theta^{-1}\left(v_{i}-\ell_{i} v\right)=\theta^{-1}\left(v_{i}-s \lambda \varphi \psi_{i} v\right)$, we get

$$
\theta^{2}\left(|\nabla z|^{2}+s^{2} \lambda^{2} \varphi^{2}|z|^{2}\right) \leq C\left(|\nabla v|^{2}+s^{2} \lambda^{2} \varphi^{2}|v|^{2}\right) .
$$

Therefore, it follows from (3.24) that

$$
\begin{aligned}
& \mathbb{E} \int_{Q}\left(s^{3} \lambda^{4} \varphi^{3}|z|^{2}+s \lambda \varphi|\nabla z|^{2}\right) d x d t \\
\leq & C \mathbb{E} \int_{Q} \theta^{2}\left(\left|b_{1}\right|^{2}|\nabla z|^{2}+b_{2}^{2}|z|^{2}+b_{3}^{2}|Z|^{2}\right) d x d t+C \mathbb{E} \int_{0}^{T} \int_{\Gamma_{0}} \theta^{2} s \lambda \varphi\left|\frac{\partial z}{\partial \nu}\right|^{2} d \Gamma d t \\
& +C \mathbb{E} \int_{Q} \theta^{2}\left(s^{2} \lambda^{2} \varphi^{2}|Z|^{2}+|\nabla Z|^{2}\right) d x d t .
\end{aligned}
$$

Taking $\lambda_{1}=\lambda_{0}$ and $s_{1}=\max \left(s_{0}, C r_{1}\right)$, and utilizing the inequality (3.26), we conclude the desired inequality (3.3). 


\section{Proof of Theorem 1.1}

In this section, we prove Theorem 1.1 by virtue of Theorem 3.1 .

Proof of Theorem 1.1. By means of the definition of $\ell$ and $\theta$ (see (3.2)), it holds that

$$
\begin{gathered}
\mathbb{E} \int_{Q} \theta^{2}\left(\varphi^{3}|z|^{2}+\varphi|\nabla z|^{2}\right) d x d t \\
\geq \min _{x \in \bar{G}}\left(\varphi\left(\frac{T}{2}, x\right) \theta^{2}\left(\frac{T}{4}, x\right)\right) \mathbb{E} \int_{\frac{T}{4}}^{\frac{3 T}{4}} \int_{G}\left(|z|^{2}+|\nabla z|^{2}\right) d x d t \\
\mathbb{E} \int_{Q} \theta^{2}\left(\varphi^{2}|Z|^{2}+|\nabla Z|^{2}\right) d x d t \\
\leq \max _{(x, t) \in \bar{Q}}\left(\varphi^{2}(t, x) \theta^{2}(t, x)\right) \mathbb{E} \int_{Q}\left(|Z|^{2}+|\nabla Z|^{2}\right) d x d t
\end{gathered}
$$

and that

$$
\mathbb{E} \int_{0}^{T} \int_{\Gamma_{0}} \theta^{2} \varphi\left|\frac{\partial z}{\partial \nu}\right|^{2} d \Gamma d t \leq \max _{(x, t) \in \bar{Q}}\left(\varphi(t, x) \theta^{2}(t, x)\right) \mathbb{E} \int_{0}^{T} \int_{\Gamma_{0}}\left|\frac{\partial z}{\partial \nu}\right|^{2} d \Gamma d t
$$

From (3.3) and (4.1)-(4.3), we deduce that

$$
\begin{aligned}
& \mathbb{E} \int_{\frac{T}{4}}^{\frac{3 T}{4}} \int_{G}\left(|z|^{2}+|\nabla z|^{2}\right) d x d t \\
\leq & C r_{1} \frac{\max _{(x, t) \in \bar{Q}}\left(\varphi^{2}(t, x) \theta^{2}(t, x)\right)}{\min _{x \in \bar{G}}\left(\varphi\left(\frac{T}{2}, x\right) \theta^{2}\left(\frac{T}{4}, x\right)\right)} \\
& \times\left\{\mathbb{E} \int_{Q}\left(|Z|^{2}+|\nabla Z|^{2}\right) d x d t+\mathbb{E} \int_{0}^{T} \int_{\Gamma_{0}}\left|\frac{\partial z}{\partial \nu}\right|^{2} d \Gamma d t\right\} \\
\leq & e^{C r_{1}}\left\{\mathbb{E} \int_{Q}\left(|Z|^{2}+|\nabla Z|^{2}\right) d x d t+\mathbb{E} \int_{0}^{T} \int_{\Gamma_{0}}\left|\frac{\partial z}{\partial \nu}\right|^{2} d \Gamma d t\right\} .
\end{aligned}
$$

Utilizing (4.4) and (2.7), we obtain that

$$
\begin{aligned}
& \mathbb{E} \int_{G}\left(\left|z_{T}\right|^{2}+\left|\nabla z_{T}\right|^{2}\right) d x \\
\leq & e^{C r_{1}}\left\{\mathbb{E} \int_{Q}\left(|Z|^{2}+|\nabla Z|^{2}\right) d x d t+\mathbb{E} \int_{0}^{T} \int_{\Gamma_{0}}\left|\frac{\partial z}{\partial \nu}\right|^{2} d \Gamma d t\right\},
\end{aligned}
$$

which concludes Theorem 1.1 immediately. 


\section{Proof of Theorem $1.2-1.3$}

This section is addressed to proofs of Theorem 1.2, 1.3,

Proof of Theorem 1.2: Since the system (1.2) is linear, we only need to show that the attainable set $A_{T}$ at time $T$ with initial datum $y(0)=0$ is $L_{\mathcal{F}_{T}}^{2}\left(\Omega ; H^{-1}(G)\right)$, that is, for any $y_{1} \in L_{\mathcal{F}_{T}}^{2}\left(\Omega ; H^{-1}(G)\right)$, we can find a pair of control

$$
(u, g) \in L_{\mathcal{F}}^{2}\left(0, T ; L^{2}\left(\Gamma_{0}\right)\right) \times L_{\mathcal{F}}^{2}\left(0, T ; H^{-1}(G)\right)
$$

such that the solution to the system (1.2) with $y(0)=0$ satisfies that $y(T)=y_{1}$ in $L_{\mathcal{F}_{T}}^{2}\left(\Omega ; H^{-1}(G)\right)$. We achieve this goal by duality argument.

Let

$$
b_{1}=-a_{1}, \quad b_{2}=-\operatorname{div}\left(a_{1}\right)+a_{2}, b_{3}=-a_{3}
$$

in the equation (1.4). We set

$$
\begin{gathered}
\mathcal{X} \triangleq\left\{\left(\left.\frac{\partial z}{\partial \nu}\right|_{\Gamma_{0}}, Z\right) \mid(z, Z)\right. \text { solves the equation (1.4) with some } \\
\left.z_{T} \in L^{2}\left(\Omega, \mathcal{F}_{T}, P ; H_{0}^{1}(G)\right)\right\} .
\end{gathered}
$$

Clearly, $\mathcal{X}$ is a linear subspace of $L_{\mathcal{F}}^{2}\left(0, T ; L^{2}\left(\Gamma_{0}\right)\right) \times L_{\mathcal{F}}^{2}\left(0, T ; H_{0}^{1}(G)\right)$. Let us define a linear functional $\mathcal{L}$ on $\mathcal{X}$ as follows:

$$
\mathcal{L}\left(\left.\frac{\partial z}{\partial \nu}\right|_{\Gamma_{0}}, Z\right)=\mathbb{E}\left\langle y_{1}, z_{T}\right\rangle_{H^{-1}(G), H_{0}^{1}(G)}-\mathbb{E} \int_{0}^{T} \int_{G} z f d x d t .
$$

From Theorem 1.1, we see that $\mathcal{L}$ is a bounded linear functional on $\mathcal{X}$. By means of the Hahn-Banach theorem, $\mathcal{L}$ can be extended to be a bounded linear functional on the space $L_{\mathcal{F}}^{2}\left(0, T ; L^{2}\left(\Gamma_{0}\right)\right) \times L_{\mathcal{F}}^{2}\left(0, T ; H^{-1}(G)\right)$. For simplicity, we still use $\mathcal{L}$ to denote this extension. Now, by Riesz Representation theorem, we know that there is a pair of random fields

$$
(u, g) \in L_{\mathcal{F}}^{2}\left(0, T ; L^{2}\left(\Gamma_{0}\right)\right) \times L_{\mathcal{F}}^{2}\left(0, T ; H^{-1}(G)\right)
$$

such that

$$
\begin{aligned}
& \mathbb{E}\left\langle y_{1}, z_{T}\right\rangle_{H^{-1}(G), H_{0}^{1}(G)}-\mathbb{E} \int_{0}^{T} \int_{G} z f d x d t \\
= & \mathbb{E} \int_{0}^{T} \int_{\Gamma_{0}} \frac{\partial z}{\partial \nu} u d \Gamma d t+\mathbb{E} \int_{0}^{T}\langle g, Z\rangle_{H^{-1}(G), H_{0}^{1}(G)} d t .
\end{aligned}
$$

We claim that this pair of random fields $(u, g)$ is the control we need. In fact, from the definition of the solution to (1.2), we have

$$
\begin{aligned}
& \mathbb{E}\left\langle y(T), z_{T}\right\rangle_{H^{-1}(G), H_{0}^{1}(G)} \\
= & \mathbb{E} \int_{0}^{T} \int_{G} z f d x d t+\mathbb{E} \int_{0}^{T} \int_{\Gamma_{0}} \frac{\partial z}{\partial \nu} u d \Gamma d t+\mathbb{E} \int_{0}^{T}\langle g, Z\rangle_{H^{-1}(G), H_{0}^{1}(G)} d t .
\end{aligned}
$$


From (5.1) and (5.2), we see

$$
\mathbb{E}\left\langle y_{1}, z_{T}\right\rangle_{H^{-1}(G), H_{0}^{1}(G)}=\mathbb{E}\left\langle y(T, \cdot), z_{T}\right\rangle_{H^{-1}(G), H_{0}^{1}(G)} .
$$

Since $z_{T}$ can be arbitrary element in $L_{\mathcal{F}_{T}}^{2}\left(\Omega ; H_{0}^{1}(G)\right)$, from the equality (15.3), we get $y(T)=y_{1}$ in $H^{-1}(G), P$-a.s.

At last, we prove Theorem 1.3. In order to present the key idea in the simplest way, we only consider a very special case of the system (1.2), that is, $a_{1}=0, a_{2}=0$ and $a_{3}=1$. The argument for the general case is very similar.

Proof of Theorem 1.3: Let us assume that $u \equiv 0$. In this case, the system (1.2) is

$$
\begin{cases}i d y+\Delta y d t d t=(y+g) d B(t) & \text { in }(0, T) \times G \\ y(t, 0)=0 & \text { on }(0, T) \times \Gamma_{0} \\ y(0)=y_{0} & \text { in } G .\end{cases}
$$

Since the system (5.4) is linear, we only need to show that the attainable set $A_{T}$ at time $T$ for the initial datum $y_{0}=0$ is not $L_{\mathcal{F}_{T}}^{2}\left(\Omega ; H^{-1}(G)\right)$. The solution of the system (5.4) is

$$
y(T)=S(T) y_{0}-i \int_{0}^{T} S(T-s)[y(s)+g(s)] d B(s) .
$$

Here $\{S(t)\}_{t \geq 0}$ is the semigroup generated by the following operator

$$
\left\{\begin{array}{l}
D(A)=H^{2}(G) \cap H_{0}^{1}(G), \\
A \varphi=i \Delta \varphi, \quad \forall \varphi \in D(A) .
\end{array}\right.
$$

From (5.5), we find $\mathbb{E}(y(T))=\mathbb{E}\left(S(T) y_{0}\right)$. Thus, if we choose a $y_{1} \in L_{\mathcal{F}_{T}}^{2}\left(\Omega ; H^{-1}(G)\right)$ such that $\mathbb{E}\left(y_{1}\right) \neq 0$, then $y_{1}$ is not in $A_{T}$, which completes the proof.

\section{Further comments and open problems}

There are plenty of open problems in the topic of this paper. Some of them are particularly relevant and could need important new ideas and further developments:

\section{- Null and approximate controllability for stochastic Schröding equations}

In this paper, we study the exact controllability for stochastic Schrödinger equations. As immediate consequences, we can obtain the null and approximate controllability for the same system. However, in order to get these two kinds of controllability, we have no reasons to use two controls. By the proof of Theorem 1.3, we know that it is not enough to put one control in the diffusion term to get the null or approximate controllability. On the other hand, suggested by the result in [17], we believe one boundary control in the drift term can guarantee the null and approximate controllability of (1.2). If we want to prove this by following the method in this paper, we will meet some essential 
difficulty. For example, to get the null controllability, we should prove the following inequality for the solution to (1.4)

$$
\mathbb{E}|z(0)|_{H_{0}^{1}(G)}^{2} \leq C \int_{0}^{T} \int_{\Gamma_{0}}\left|\frac{\partial z}{\partial \nu}\right|^{2} d \Gamma d t .
$$

However, if we utilize the method in this paper, we only get

$$
\mathbb{E}|z(0)|_{H_{0}^{1}(G)}^{2} \leq C \int_{0}^{T} \int_{\Gamma_{0}}\left|\frac{\partial z}{\partial \nu}\right|^{2} d \Gamma d t+\int_{0}^{T}|Z|_{H_{0}^{1}(G)}^{2} d t .
$$

There is an additional term containing $Z$ in the right hand side. This terms comes from the fact that, in the Carleman estimate, we regard $Z$ as an nonhomogeneous term rather than part of the solution. Hence, it has to appear in the right hand side of the inequality. Therefore, we believe that one should introduce some new technique, for example, a Carleman estimate in which the fact $Z$ is part of the solution is involved, to get rid of the additional term containing $Z$. However, we do not know how to achieve this goal now.

\section{- Exact controllability for stochastic Schrödinger equations with less restric- tive condition}

In this paper, we get the exact controllability for (1.2) for $\Gamma_{0}$ given by (1.1). It is well known that a sharp sufficient condition for exact controllability for deterministic Schrödinger equations in analytic domain with time invariant lower order terms is that the triple $\left(G, \Gamma_{0}, T\right)$ satisfies the Geometric Optic Condition introduced in [2] (see [14] for example). It would be quite interesting and challenging to extend this result to the stochastic setting, but it seems that there are lots of things should be done before solving this problem. For instance, the main idea in [14] is as follows:

Based on a diadic decomposition of the Fourier representation of solutions of the Schrödinger equation, the author show that, to some extent, one can regards them as superposition of an infinite sequence of solutions of wave equations with velocity of propagation tending to infinity. Then, the fact that the Geometric Optic Condition is satisfied for some finite time $T$ suffices for the exact controllability of the Schrödinger equation to hold for all $T>0$.

If one follow the above idea, then propagation of singularities for stochastic partial differential equations, at least, for stochastic hyperbolic equations, should be established. However, as far as we know, this topic is completely open.

Further, there are some results showing that, in some situations in which the Geometric Optic Condition is not fulfilled in any time $T$, one can still obtain exact controllability for the Schrödinger equation. For instance, in [9], it is showed that, when the domain $G$ is a square, for any open non-empty subset $G_{0}$ of $G$, the exact controllability of the Schrödinger equation holds in any time $T$, in the space $L^{2}(G)$ and with internal controls in $L^{2}\left((0, T) \times G_{0}\right)$. How to prove such kind of result is very interesting. 


\section{References}

[1] A. Al-Hussein, Strong, mild and weak solutions of backward stochastic evolution equations. Random Oper. Stochastic Equations. 13(2005), no. 2, 129-138.

[2] C. Bardos, G. Lebeau and J. Rauch, Sharp sufficient conditions for the observation, control and stabilizion of waves from the boundary, SIAM J. Control Optim. 30(1992), 1024-1065.

[3] K. Beauchard and C. Laurent,Local controllability of $1 D$ linear and nonlinear Schrödinger equations with bilinear control. J. Math. Pures Appl. 94(2010), no. 5, 520-554.

[4] J. Bourgain, N. Burq and M. Zworski, Control for Schrödinger equations on 2-tori: rough potentials. http://arxiv.org/pdf/1301.1282.pdf

[5] N. Burq and M. Zworski, Geometric control in the presence of a black box. J. Amer. Math. Soc. 17 (2004), no. 2, 443-471.

[6] C. Cazacu, Schrödinger operators with boundary singularities: Hardy inequality, Pohozaev identity and controllability results. J. Funct. Anal. 263(2012), no. 12, 3741-3783.

[7] G. Da Prato and J. Zabczyk, Stochastic equations in infinite dimensions. Encyclopedia of Mathematics and its Applications, 44. Cambridge University Press, Cambridge, 1992.

[8] S. Ervedoza and J.-P. Puel, Approximate controllability for a system of Schrödinger equations modeling a single trapped ion. Ann. Inst. H. Poincaré Anal. Non Linéaire 26(2009), no. 6, 2111-2136.

[9] S. Jaffard, Contrôle interne exact des vibrations d'une plaque carrée. C. R. Acad. Sci. Paris Sér. I Math. 307(1988), no. 14, 759-762.

[10] V. Komornik, Exact controllability and stabilization. The multiplier method. RAM: Research in Applied Mathematics. Masson, Paris; John Wiley \& Sons, Ltd., Chichester, 1994.

[11] V. Komornik and P. Loreti, Fourier series in control theory. Springer Monographs in Mathematics. Springer-Verlag, New York, 2005.

[12] I. Lasiecka, J.-L. Lions and R. Triggiani, Nonhomogeneous boundary value problems for second order hyperbolic operators. J. Math. Pures Appl. 65(1986), no. 2, 149-192.

[13] I. Lasiecka, R. Triggiani and X. Zhang, Global uniqueness, observability and stabilization of nonconservative Schrödinger equations via pointwise Carleman estimates: Part I. $H^{1}$-estimates. J. Inv. Ill-posed Problems. 11(2004), 43-123.

[14] G. Lebeau, Contrôle de l'équation de Schrödinger. J. Math. Pures Appl. 71(1992), no. 3, 267-291.

[15] J.-L. Lions and E. Magenes, Non-homogeneous boundary value problems and applications. Vol. I. Translated from the French by P. Kenneth. Die Grundlehren der mathematischen Wissenschaften, Band 181. Springer-Verlag, New York-Heidelberg, 1972.

[16] J.-L. Lions and E. Magenes, Non-homogeneous boundary value problems and applications. Vol. II. Translated from the French by P. Kenneth. Die Grundlehren der mathematischen Wissenschaften, Band 182. Springer-Verlag, New York-Heidelberg, 1972.

[17] Q. Lü, Some results on the controllability of forward stochastic heat equations with control on the drift. J. Funct. Anal. 260(2011), no. 3, 832-851.

[18] Q. Lü,Observability estimate for stochastic Schrödinger equations and its applications. SIAM J. Control Optim. 51(2013), no. 1, 121-144.

[19] N. I. Mahmudov and M. A. McKibben, On backward stochastic evolution equations in Hilbert spaces and optimal control. Nonlinear Anal. 67(2007), no. 4, 1260-1274.

[20] E. Machtyngier, Exact controllability for the Schrödinger equation, SIAM J. Control Optim. 32(1994), $24-34$. 
[21] S. Peng, Backward stochastic differential equation and exact controllability of stochastic control systems. Progr. Natur. Sci. (English Ed.) 4(1994), no. 3, 274-284.

[22] L. Rosier and B. Zhang, Exact boundary controllability of the nonlinear Schrödinger equation. J. Differential Equations 246(2009), no. 10, 4129-4153.

[23] X. Zhang, Explicit observability estimate for the wave equation with potential and its application, $R$. Soc. Lond. Proc. Ser. A Math. Phys. Eng. Sci., 456(2000), 1101-1115.

[24] E. Zuazua, Remarks on the controllability of the Schrödinger equation, Quantum control: mathematical and numerical challenges, 193-211, CRM Proc. Lecture Notes, 33, Amer. Math. Soc., Providence, RI, 2003. 